\title{
Mindfulness y rendimiento deportivo
}

\author{
Israel Mañas*, Jesús del Águila, Clemente Franco, Mª Dolores Gil y Consolación Gil \\ Universidad de Almería
}

(Recibido 14 abril 2014; Aceptado 25 abril 2014)

RESUMEN: La inclusión del mindfulness en el deporte es un campo reciente. Mientras que la psicología del deporte ha dependido principalmente de la "segunda ola" de intervenciones cognitivo-conductuales durante las últimas cuatro décadas, una nueva aproximación que incluye al mindfulness se ha desarrollado recientemente: la "tercera ola". Esta nueva aproximación asume la idea de que el rendimiento es un estado que no se basa en el auto-control o cambio del comportamiento, sino que es un estado que emerge del reconcomiendo y aceptación de los pensamientos, emociones y sensaciones corporales. La práctica del mindfulness permite aprender a observar y aceptar los pensamientos, emociones y sensaciones corporales sin intentar eliminarlos o modificarlos. Este trabajo revisa los dos principales programas de mindfulness para el rendimiento deportivo, ambos de la "tercera ola": el Mindful Sport Performance Enhancement (MSPE) y el Mindfulness-Acceptance-Commitment (MAC).

Palabras clave: mindfulness, atención plena, rendimiento deportivo, atletas.

\section{Mindfulness and sport performance}

\begin{abstract}
Mindfulness in sports is a recent field. While sport psychology relied mainly on "second wave" cognitive-behavioural interventions for the last four decades, a new approach has recently been developed in sport psychology including mindfulness: a "third wave" approach. This new approach assumes that ideal performance is a state that is not based on self-control or change in behaviour, but rather a state that arises from recognition and acceptance of thoughts, emotions and bodily sensations. Practicing mindfulness allows learns to observe and accept the thoughts, emotions, and body sensations, without making any attempt to eliminate or modify them. This paper reviews the main programs of mindfulness in sport performance both from the "third wave": Mindful Sport Performance Enhancement (MSPE) and Mindfulness-Acceptance-Commitment (MAC).
\end{abstract}

Keywords: mindfulness, sport performance, athletes.

\section{INTRODUCCIÓN}

En el contexto de la actividad física y el deporte, especialmente para los atletas y deportistas de competición o alto nivel, uno de los objetivos fundamentales es el incremento del rendimiento deportivo (Gooding y Gardner, 2009). Cuando se habla de mejorar el rendimiento deportivo, se suelen tener en cuenta variables 
y demandas de tipo fisiológicas, sin embargo, el deporte es de carácter multidimensional, requiere de habilidades psicológicas para la mejora y optimización del rendimiento (Birrer y Morgan, 2010). El rendimiento óptimo se ha descrito como "la correcta combinación de las condiciones cognitivas, afectivas y fisiológicas que permiten adecuadamente que las habilidades aprendidas ocurran de una manera aparentemente sin esfuerzo y automática" (Gardner y Moore, 2007, p.4).

Durante décadas, la psicología del deporte ha investigado el clima o estado mental ideal que favorezca que los deportistas y atletas alcancen su mejor rendimiento (Williams y Straub, 2010) esto incluye, por supuesto, la adquisición y desarrollo de ciertas habilidades psicológicas. Para adquirir y desarrollar estas habilidades la psicología aplicada al deporte se ha nutrido principalmente de técnicas y herramientas que provienen, de lo que en psicología se denomina genéricamente la tradicional aproximación cognitiva-conductual. Decimos "tradicional" porque esta aproximación se corresponde con la denominada "segunda ola de terapias" y a las terapias dentro de este movimiento se las conoce como "terapias de segunda generación"; en contraste con la "tercera ola de terapias" y las "terapias de tercera generación", entre las que se enmarcamos el mindfulness o atención plena (e.g., véase Hayes, 2004; Mañas, 2007).

Una de limitaciones más importantes de las terapias de segunda generación son los datos experimentales que indican precisamente que los intentos de control, reducción o eliminación de los eventos privados o internos (e.g., pensamientos, emociones, sensaciones corporales, etc.), justamente objetivos de intervención explícitos de la tradicional aproximación cognitivo-conductual, producen paradójicamente, y en muchos casos, efectos contrarios a los deseados. Entre estos efectos se han descrito notables incrementos en la intensidad, la frecuencia, así como en la duración, e incluso, en la accesibilidad a los eventos privados no deseados (para más detalle véase Purdon, 1999; Rassin, Merckelbach y Muris, 2000). Esto datos, suponen un claro desafío a los principios y asunciones de las terapias de segunda generación: la necesidad de cambiar los eventos internos (e.g., pensamientos, emociones y sensaciones corporales).

Este trabajo invita a considerar al mindfulness como una alternativa al control de los eventos privados en el contexto de la actividad física y el deporte. Por otro lado, este trabajo revisa los objetivos y técnicas tradicionales ("segunda ola") para incrementar el rendimiento deportivo y los programas de mindfulness ("tercera ola") que se utilizan actualmente con el mismo objetivo: el programa Mindful Sport Performance Enhancement (MSPE) y el programa MindfulnessAcceptance-Commitment (MAC).

\section{OBJETIVOS Y TÉCNICAS TRADICIONALES PARA MEJORAR EL RENDI- MIENTO DEPORTIVO}

La tradicional aproximación cognitivo-conductual en psicología aplicada al deporte ("segunda ola") se basa en la asunción de que la reducción de los estados 
internos negativos resulta necesaria para incrementar las emociones positivas y los niveles de confianza, por lo que consecuentemente se mejorará el rendimiento atlético (Hardy, Jones y Gould, 1996). Esta asunción ha promovido el entrenamiento sistemático de ciertas habilidades en los atletas con el objetivo primordial de proveerles de estrategias de control para que eliminen o cambien los estados internos valorados como negativos con la esperanza de que esto contribuya a mejorar su nivel de rendimiento. Por ejemplo, a un atleta se le puede entrenar para que se relaje en momentos que experimente ansiedad, para que elimine ciertos pensamientos valorados también como negativos, o por ejemplo, para cambiar o alterar ciertas emociones o sentimientos que puedan emerger. De forma resumida podríamos decir que a los atletas se les hace creer que tienen la necesidad de cambiar y se les enseña a hacerlo, ciertos pensamientos, emociones y sensaciones físicas con el objetivo de realizar una correcta ejecución o mejorar su rendimiento deportivo.

Las principales habilidades y técnicas que se les enseña a los atletas son: (1) la imaginería; (2) el control del aurosal; (3) la modificación de auto-verbalizaciones; y (4) otras rutinas pre-competición para mejorar los procesos de autoregulación que se entienden ideales para alcanzar el mejor rendimiento (para más detalle véase Schwanhausser, 2009). El uso de estas habilidades mentales con los atletas primeramente se inspiró en la teoría cognitiva social de Bandura (1977) y en el desarrollo de la modificación cognitiva-conductual de Meichenbaum (1977).

El desarrollo de estas habilidades ha dominado la psicología aplicada al deporte durante décadas. Estas técnicas representan los métodos o herramientas principales para ayudar a los atletas a mejorar su rendimiento deportivo. Sin embargo, existen diferentes modelos y teorías que sostienen la noción de que no es necesario modificar los estados internos para conseguir el estado ideal para el rendimiento. Por ejemplo, modelos como el CUSP Catastrople Model, el Individual Zones of Optimal Functioning (IZOF) model y la Processing Efficiency Theroy (PET) mantienen que el estado ideal para alcanzar el máximo y óptimo rendimiento puede ocurrir a pesar o mientras se experimentan pensamientos, sensaciones o emociones negativas (para una discusión más detallada véase Mellalieu, Hanton y Fletcher, 2006). Por ejemplo la PET (Eysenck y Calvo, 1992) sostiene que cuando los componentes cognitivos de la ansiedad se manifiestan, la eficiencia en procesar la información puede verse mermada significativamente a través de una reducción de la capacidad de la memoria de trabajo (working memory) debido a que el foco de la atención de ha desviado desde la tarea relevante (la ejecución deportiva, en este caso) a otra irrelevante (e.g., eliminar un pensamiento negativo).

Parece lógico considerar que si un atleta tiene que controlar ciertos pensamientos, emociones o sensaciones corporales, valorados como negativos o contraproducentes, especialmente si cree que ha de hacerlo para que su rendimiento deportivo sea el óptimo, deba invertir energía y tiempo en ejercer el control necesario para conseguirlo. La primera consecuencia que se deriva de esto es que si el 
atleta ha de invertir cierta cantidad de tiempo y esfuerzo en hacerlo, consecuentemente, no estará al cien por cien, esto es, plenamente atento o centrado en la propia tarea, con toda su energía puesta en la misma, con su posible repercusión negativa en su ejecución, obviamente. Por otro lado, puede ser que lo consiga o no lo consiga. Si consigue su objetivo, aparentemente todo iría bien, pero lo cierto es que esto contribuya a reforzar la creencia de que es necesario hacerlo. Por otra parte, si no lo consigue puede ser que genere una reacción emocional intensa negativa (e.g., frustración o miedo) interfiriendo a su propio rendimiento y que incluso se enzarce en una lucha intentando controlar o cambiar algo que no puede controlar ni cambiar, al menos no en ese momento.

Otra consecuencia negativa que se deriva de este planteamiento o proceder, como se ha señalado anteriormente, es que, paradójicamente, en muchos casos los intentos por controlar o eliminar ciertos eventos privados o internos valorados como negativos hacen que estos persistan y aumenten en frecuencia e intensidad. Por último, señalar que puede ocurrir que las destrezas y ejecuciones que deberían de producirse de manera automática (y que han sido entrenadas sistemáticamente una y otra vez a través del entrenamiento deportivo) podrían no realizarse de manera fluida mientras el atleta está intentando controlar algún pensamiento o emoción. Es decir, la ejecución de las rutinas entrenadas podrían no realizarse de manera óptima o automatizada e incluso verse obstaculizadas seriamente. En resumen, existen varias consecuencias negativas que se pueden derivar del hecho de querer cambiar o eliminar ciertos eventos internos en el contexto de la práctica de la actividad física y el deporte, especialmente en el deporte de alta competición donde se le otorga un valor primordial al rendimiento.

Mindfulness ("tercera ola") representa otra vía, una alternativa radicalmente diferente que implica relacionarnos con nuestros propios eventos internos de una manera completamente distinta. Mindfulness no pretende cambiarlos o eliminarlos en absoluto. Mindfulness representa un modo de relacionarnos con ellos desde la ecuanimidad, la consciencia y la aceptación radical de la experiencia. Abandonamos la lucha y el control para pasar a adoptar una postura contemplativa. Éste es uno de los modos en que el mindfulness puede contribuir de forma positiva a que los atletas aumenten su rendimiento deportivo.

\section{MINDFULNESS}

Mindfulness se suele traducir al español como Atención Plena o Conciencia Plena. Mindfulness representa el "corazón" o enseñanza central de la psicología budista. Es un estado de consciencia que implica prestar atención a la experiencia del momento presente. Se cultiva y desarrolla mediante la práctica de la meditación, a través de la cual nos volvemos menos reactivos a lo que nos ocurre en el momento actual.

Podemos considerar al mindfulness como la consciencia que emerge a través de poner atención intencional, en el momento presente, de manera no condenatoria, del flujo de la experiencia momento a momento (Kabat-Zinn, 2003). De for- 
ma general, mindfulness ha sido descrito como una clase de conciencia centrada en el presente, no elaborativa ni condenatoria, en la que cada pensamiento, sentimiento o sensación que surge en el campo atencional es reconocido y aceptado tal y como es (Segal, Williams y Teasdale, 2002).

Bishop et al. (2004) diferencian dos tipos de componentes implicados en la práctica del mindfulness: (1) la autorregulación de la atención, cuya función es el mantenimiento y la redirección de la atención incrementando el reconocimiento de los eventos mentales en el momento presente; (2) la orientación hacia la experiencia, implica adoptar una relación particular hacia la propia experiencia en el momento presente caracterizada por una actitud de curiosidad, apertura y aceptación, aprendiendo a no reaccionar automáticamente a la estimulación que se esté experimentando.

Kabat-Zinn (2003) señala siete elementos primordiales relacionados con la actitud hacia la práctica de la atención plena: no juzgar, paciencia, mente de principiante, confianza, no esforzarse, aceptación y ceder, dejar ir o soltar. Según este autor, la práctica del mindfulness nos lleva a un estado en el que se es consciente de la realidad del momento presente, aceptando y reconociendo lo que existe en el aquí y el ahora, pero sin quedar atrapado en los pensamientos o en las reacciones emocionales que la situación provoca.

\section{PROGRAMAS DE ENTRENAMIENTO EN MINDFULNESS PARA ATLETAS}

\section{Mindful Sport Performance Enhancement (MSPE)}

El programa MSPE se desarrolló en el año 2006 (Kaufman y Glass, 2006). Esta intervención proviene del programa Mindfulness-Based Stress Reduction (MBSR) de Kabat-Zinn $(1990,2003)$ y de la Mindfulness-Based Cognitive Therapy (MBCT) de Segal, Williams y Teasdale (2002) así como de otras fuentes relevantes relacionadas con el mindfulness y el deporte (e.g., Gallwey, 1974). El MSPE está estructurado para ser adaptado a cualquier deporte.

La versión inicial del MSPE fue diseñado como un protocolo de 4 semanas. Incorporaba conceptos de libros de tiro con arco (Lee y de Bondt, 2005), golf (Rotella y Cullen, 2004) y running (Dreyer y Dreyer, 2009). Por ello, atletas de estos deportes se incluyeron desde el principio en las investigaciones de este programa. Recientemente se ha desarrollado una nueva versión extendida de este programa. La versión extendida consiste en un protocolo de 6 semanas de duración. Semanalmente tiene lugar una sesión en grupo de 90 minutos. Además el programa contiene prácticas para ser realizadas en casa diariamente (Kaufman, Glass y Pineau, 2012). Se elaboraron instrucciones específicas para todos los ejercicios de mindfulness. Por otro lado, se realizaron grabaciones en CD de estos ejercicios que se facilitan para la práctica diaria en casa. El propósito del MSPE es entrenar a los atletas en los principios fundamentales para el cultivo del mindfulness, y después ayudarles a que gradualmente apliquen las habilidades 
de mindfulness tanto en sus rutinas de rendimiento deportivo así como en sus propias vidas.

En las sesiones iniciales se presenta a los atletas una orientación general y la lógica del programa, la cual incluye una explicación de qué es mindfulness, cómo el entrenamiento en mindfulness puede ser útil para los atletas y cómo las habilidades aprendidas en el programa MSPE son directamente aplicadas en su deporte.

Los ejercicios claves que contiene el protocolo son: (1) ejercicio del caramelo, una variante del ejercicio de la uva pasa de Kabat-Zinn (1990), el cual introduce el concepto de consciencia invitando a los atletas a que se focalicen y usen sus sentidos sensoriales mientras lentamente comen trozos de chocolate; (2) una meditación sentada que va incrementado su duración a lo largo del curso desde 10 a, al menos, 25 minutos, en la que a los atletas primero se les guía para que focalicen la atención en su respiración, después en las sensaciones de sus cuerpos y finalmente a los sonidos que hay a su alrededor; (3) un body-scan (escaneo o exploración corporal) en el que se le dan pautas para que pongan su atención sobre las diferentes partes de sus cuerpos de forma secuenciada desde los pies hasta la cabeza, al tiempo que se les dice que noten y acepten cualquier tipo de sensación que emerja durante el proceso; (4) mindful yoga, que incluye una serie de ejercicios básicos de posturas de yoga que permite a los atletas a practicar el mantenimiento de una consciencia mindful de sus cuerpos y mentes mientras están en movimiento; (5) una meditación caminando, en la que los atletas son guiados para que estén completamente conscientes de las sensaciones que experimentan mientras sus cuerpos lentamente se mueven y pasan de estar parados a caminar y variando los pasos; y (6) una meditación específica deportiva (e.g., meditación running), diseñada para ofrecer a los atletas la oportunidad de aplicar las habilidades de mindfulness que han desarrollado a través de las sesiones en el programa a los movimientos y sensaciones que ello experimentan cuando practican su deporte.

El objetivo de estos ejercicios centrales es enseñar progresivamente a los atletas a que pasen de una práctica sedentaria de mindfulness a una práctica activa de mindfulness. La culminación de esta progresión desde mindfulness en quietud a mindfulness en movimiento es la introducción de la meditación específica deportiva la cual pretende crear el puente necesario entre el cultivo del mindfulness y la aplicación del mindfulness durante la práctica deportiva.

Existen varios estudios que muestran la eficacia del MSPE. Dos estudios (De Petrillo, Kaufman, Glass y Arnkoff, 2009; Kaufman, Glass y Arnkoff, 2009) y una investigación con medidas de seguimiento (Thompson, Kaufman, De Petri1lo, Glass y Arnkoff, 2011a) han sido realizados empleando la versión de 4 semanas del MSPE, y un análisis controlado de la versión extendida de 6 semanas se ha realizado recientemente (Pineau, Kaufman y Glass, 2012).

Empleando una muestra de la comunidad de arqueros y golfistas, Kaufman et al. (2009) encontraron incrementos significativos en las variables de mindfulness estado y mindfulness rasgo para los golfistas, mindfulness rasgo para la mayoría 
de los arqueros y en estado flow para toda la muestra. Adicionalmente, feedback después de la impartición del programa puso de manifiesto que los atletas sentían que el MSPE tuvo un impacto significativamente positivo sobre su rendimiento y que ellos tenían expectativas de beneficios en el futuro.

De Petrillo et al. (2009) adaptaron el protocolo de 4 semanas del MSPE para corredores y encontraron un incremento significativo en el mindfulness estado y en una dimensión del mindfulness rasgo comparando las medidas pre y post intervención, además de una reducción significativa en las variables de perfeccionismo y ansiedad relacionadas con el deporte. Sin embargo, en este estudio no se hallaron significativos cambios en su rendimiento, que se analizaron comparando medidas de autoinformes de su mejor tiempo antes y después de la intervención.

Un seguimiento de un año de arqueros, golfistas y corredores que habían recibido el entrenamiento del programa MSPE en los estudios iniciales mostró que los atletas experimentaron un incremento significativo en mindfulness rasgo desde que realizaron el taller (Thompson et al., 2011). Además, tanto los golfistas como los corredores informaron que su rendimiento mejoró significativamente (i.e., puntuaciones de rondas de 18 hoyos y tiempo de millas, respectivamente) desde que terminó el taller. Sin un grupo control, es imposible decir si los cambios fueron resultado del entrenamiento suministrado por el programa MSPE por encima o por debajo del efecto de la propia práctica durante un año, es decir, debido a la experiencia desarrollada por los atletas en su deporte, ya que otros resultados sugieren esta posibilidad. Específicamente, mejoras en las puntuaciones de los golfistas fueron significativamente relacionadas con incrementos en la dimensión feedback ambiguo del flow rasgo, y la mejora del rendimiento de los corredores se asoció con incrementos en mindfulness. Estos resultados podrían resultar particularmente importantes dado que Kaufman et al. (2009) observaron un incremento significativo en la dimensión feedback ambiguo del flow estado desde el inicio (pre) hasta el después (post) de la intervención. Por otro lado, los atletas de estudios iniciales mostraban incrementos en mindfulness.

Recientemente, un estudio controlado de la versión extendida del programa MSPE ha sido llevado a cabo empleando una muestra de los corredores universitarios de larga distancia de la División I (Pineau et al., 2012). Los resultados pre-post no han sido todavía analizados, sin embargo, los primeros resultados de medidas pre intervención han revelado que, además de el número de millas corridas por semana, el componente de aceptación de mindfulness predice significativamente el tiempo corriendo.

\section{Mindfulness-Acceptance-Commitment (MAC)}

El programa MAC (Gardner y Moore, 2004, 2007) es una combinación de mindfulness y técnicas de la Acceptance and Commitment Therapy (ACT; Hayes, Strosahl y Wilson, 1999) que fue diseñado para utilizarse con personas con alto nivel de rendimiento y puede ser usado con atletas.

El MAC comparte ciertas similitudes con el concepto de flow (Csikzentmi- 
halyi, 1990) que describe el estado de ejecución ideal en los atletas como una intensificada consciencia al momento presente y concentración en la tarea. El flow también ha sido descrito como la "fusión de la acción y las consciencia" (Csikzentmihalyi, 1990). El programa MAC parece promover el estado de ejecución óptima en atletas y la experiencia de flow. Este programa semi-estructurado está organizado en 5 componentes, incluye: (1) psicoeducación; (2) mindfulness; (3) identificación de valores y compromiso; (4) aceptación; y (5) integración y práctica.

El primer componente del MAC (psicoeducación) implica proveer una presentación de la lógica teórica que usa el MAC, el establecimiento del propósito y metas del MAC y ayudar a los atletas a comprender sus experiencias de ejecución desde la perspectiva del MAC. El MAC enseña a los atletas a mantener la atención posada sin ejercer ningún control sobre sus experiencias internas. El proceso educacional del MAC puede implicar una discusión del proceso de auto-regulación y los intentos previos de los atletas en regular o controlar sus experiencias internas mientras están compitiendo. Una de las metas u objetivos del programa MAC es ayudar a los atletas a desarrollar la habilidad de permitir que las experiencias se sucedan naturalmente, tales como emociones y pensamientos para ser reconocidos como eventos que son transitorios de la experiencia humana. Mindfulness puede resultar extremadamente útil para alcanzar este objetivo (Gardner y Moore, 2007).

El segundo componente del programa MAC (mindfulness) consiste en enseñar a los atletas técnicas de mindfulness para que lleguen a ser más conscientes de sus experiencias internas y externas. Más importante, el atleta llegará a ser capaz de centrar su atención en el momento presente reconociendo su experiencia de modo no juicioso ni evaluativo. Los atletas aprenderán a simplemente observar y "dejar ir" cualquier pensamiento negativo o desagradable o emoción sin tratarlos como entidades reales que requieren que actuemos en su contra. Este es el comienzo del proceso de defusión cognitiva (cognitive defusion) que ayudará a los atletas en su habilidad para llegar a focalizarse sobre la tarea. La defusión cognitiva es el proceso que desmonta o rompe aprendizajes por asociaciones entre pensamientos, sentimientos y comportamientos, de tal manera que los atletas no responderán ante sus estados internos como entidades reales o realidades.

Una vez que los atletas tienen una comprensión general de programa MAC y son iniciados en los ejercicios de mindfulness, estarán preparados para identificar qué valores (tercer componente, identificación de valores y compromiso) relacionados con sus rendimiento son importantes para ellos. La actuación acorde a los valores resulta esencial de cara a las acciones que se hacen día a día y estimula al atleta a vivir una vida dirigida por sus valores en lugar de una vida dirigida por una emoción que se limita exclusivamente a una reacción aislada.

El cuarto componente (aceptación) implicar invitar a los atletas a que comparen el coste de vivir acorde a los valores de la vida de uno con los esfuerzos por controlar o evitar sus estados internos. Se anima a los atletas a que dejen de evitar su experiencia y que pasen a aceptarla y que se aproximen a la misma sin 
juzgarla. A través de la aceptación de los estados internos, los atletas llegarán a ser capaz de "dejar ir" cualquier emoción, pensamiento o sensación corporal y a tener la habilidad de focalizar su atención sobre la ejecución de la tarea.

El quinto componente (integración práctica) se centra en la integración y mejora del mindfulness, aceptación y compromiso. Además, se examinan posibles obstáculos potenciales para el mantenimiento sucesivo de las habilidades adquiridas.

Existen varios estudios que muestran la eficacia del MAC. Wolanin (2005) investigó la versión preliminar del protocolo MAC con 11 estudiantes universitarios de la Division I. Basados en medidas de clasificaciones, los atletas del grupo experimental mostraron mejoras significativas en su rendimiento atlético en comparación con el grupo control.

El estudio de caso de Lutkenhouse (2007) examinó el impacto del programa MAC con una jugadora de lacrosse de 19 años que fue clasificada como Performance Dysfunction y quien había experimentado significativas dificultades en su regulación emocional. Desde el programa no se intentó realizar un formal estudio de caso, los resultados se basaron en el Wolanin's Sport Perfomance Questionnaire y observaciones conductuales. El programa o intervención fue exitoso, pero tras un significativo número de ensayos y entrenamiento.

Lutkenhouse, Gardner y Morrow (2007) realizaron un estudio controlado aleatorizado con 118 atletas universitarios de la Division I para investigar el programa MAC en comparación con protocolo tradicional de intervención en entrenamiento de habilidades de ejecución. Los resultados mostraron mejoras significativas en el rendimiento atlético en las medidas postest en el grupo MAC en comparación con el grupo que empleó el protocolo tradicional. Además, el grupo MAC mostró significativas reducciones en agresividad y evitación experiencial e incrementos significativos en las experiencias de flow (Gardner y Moore, 2007; Lutkenhouse et al., 2007).

Más recientemente, Schwanhausser (2009), en otro estudio de caso, examinó el impacto de un programa MAC con adolescentes saltadores de trampolín. Los resultados indicaron cambios significativos en el rendimiento atlético a través de medidas objetivas de rendimiento en salto, así como también incrementos en mindfulness, experiencias de aceptación y de estados de flow.

\section{CONCLUSIÓN}

El objetivo del mindfulness no es el control o supresión de los pensamientos, emociones o sensaciones corporales, más bien es la aceptación de los mismos experimentando y comprendiendo que son eventos transitorios. Esto contrasta de forma directa con el principal objetivo de las tradicionales técnicas empleadas para que los atletas mejoren su rendimiento deportivo (e.g., controlar la ansiedad, eliminar pensamientos negativos, cambiar el estado de ánimo, etc.).

La persona que practica mindfulness aprende a observar y aceptar los pensamientos, las sensaciones y las emociones que experimenta. A través de la obser- 
vación se desidentifica, genera perspectiva y los trasciende. En cierto sentido, se libera del contenido de los mismos. El practicante no intenta eliminar, suprimir, reducir o alterar de ningún modo sus pensamientos, emociones y sensaciones corporales ya que los intentos de control en ocasiones generan más problemas y sufrimiento (Campbell, Barlow, Brown y Hoffman, 2006).

Mindfulness nos permite contemplar los pensamientos, emociones y sensaciones que experimentamos como eventos que fluyen de forma continua y cambiante. Observándolos, siendo conscientes de su carácter transitorio e impermanente, abandonamos el patrón habitual de juzgarlos y considerarlos como "cosas" que poseen entidad propia, pues aprendemos a observarlos sin reaccionar necesariamente ante ellos y generamos sabiduría en cuanto a su carácter efímero e impermanente (Kabat-Zinn, 1990, 2003). Consecuentemente, aprendemos a ser más conscientes de los pensamientos y las emociones que tienen lugar en cada momento, cambiando nuestra forma de relacionarnos y de reaccionar ante ellos. De este modo, los pensamientos y los sentimientos pasan a contemplarse como eventos mentales transitorios que no tienen porque reflejar la realidad del momento presente.

Un atleta mindful podrá desarrollar consciencia en torno a sus propios pensamientos, emociones y sensaciones corporales y se relacionará con ellos de una manera más sabía y llena de consciencia. Esta relación está caracterizada por una aceptación radical y profunda de su propia experiencia. Implica ser consciente a cada momento no sólo de su propia experiencia sino también de lo que está ocurriendo a su alrededor. Por ello, no ha de perder tiempo en controlar nada (que no sea la propia atención) y no ha de invertir tiempo y energía (en ocasiones luchando duramente) para eliminar ni cambiar nada que esté valorando negativamente (ya que también aprende a no juzgar la experiencia y a no reaccionar ante ella ni contra ella). Consecuentemente, estará más focalizado en la tarea, podrá responder o realizar su ejecución con mayor precisión y exactitud, sufrirá menos distracciones y el aprendizaje de lo entrenado y aprendido podrá fluir de forma libre y automática de forma beneficiosa.

Por otro lado, los datos expuestos en este trabajo ponen de manifiesto que tanto el programa MSPE como el programa MAC pueden resultar útiles para incrementar el rendimiento deportivo. El elemento clave a destacar es que estos programas no pretenden entrenar a los atletas a que controlen, eliminen o alteren de ningún modo sus propios estados internos y experiencias (e.g., pensamientos, emociones, sensaciones corporales, etc.) como sí es el caso de la mayoría de las intervenciones que se han realizado y se realizan actualmente (intervenciones cognitivo-conductuales tradicionales de segunda generación). El objetivo de estos programas (intervenciones de tercera generación) es muy diferente, consiste en enseñar a los atletas a que dejen de controlar y a que practiquen la aceptación de tales eventos desarrollando conciencia y sabiduría en torno a los mismos, comprendiendo y experimentando que son eventos pasajeros o transitorios. De esta manera, los atletas aprenden a tomar distancia con respecto a sus propios eventos privados o internos (e.g., pensamientos, sensaciones físicas, emociones) 
al tiempo que se desidentifican y generan flexibilidad cognitiva. Al no tener que gastar tiempo ni energía en controlarlos, la capacidad de estar focalizado en la tarea no se ve mermada y la ejecución y rendimiento se mantiene o aumenta.

En definitiva, debido a las consecuencias negativas que pueden derivarse de tener como objetivo de intervención el controlar o cambiar lo que se está experimentado, en el contexto del deporte, y especialmente para los atletas o competidores de alto nivel, merece la pena abogar por la práctica del mindfulness, la cual no implica el control sino podríamos decir "gestión o aceptación consciente" de nuestros eventos privados.

\section{REFERENCIAS}

Bandura, A. (1977). Self-efficacy: Towards a unifying theory of behavioral change. Psychological Review, 84, 192-215.

Birrer, D. y Morgan, G. (2010). Psychological skills training as a way to enhance an

Bishop, S. R., Lau, M., Shapiro, S., Carlson, L., Anderson, N., Cardomy, J., Segal, Z., Abbey, S., Speca, M., Velting, D. y Devins, G. (2004). Mindfulness: A proposed operational definition. Clinical Psychology: Science and Practice, 10, 230-241.

Csikszentmihalyi, M. (1990). Flow: The psychology of optimal experience. New York: Harper \& Row.

De Petrillo, L. A., Kaufman, K. A., Glass, C. R. y Arnkoff, D. B. (2009). Mindfulness for longdistance runners: An open trial using Mindful Sport Performance Enhancement (MSPE). Journal of Clinical Sport Psychology, 4, $357-$ 376.

Dreyer, D. y Dreyer, K. (2009). ChiRunning: A revolutionary approach to effortless, injury-free running (Rev. ed.). New York: Simon \& Schuster.

Eysenck, M. W. y Calvo, M. G. (1992). Anxiety and performance: The processing efficiency theory. Cognition and Emotion, 6, 409-434.

Gallwey, W. T. (1974). The inner game of tennis. New York: Random House.

Gardner, F. L. y Moore, Z. E. (2004). A mindfulness-acceptance-commitmentbased approach to athletic performance enhancement: Theoretical considerations. Behavior Therapy, 35, 707-723.

Gardner, F. L. y Moore, Z. E. (2007). The psychology of enhancing human performance: The mindfulness-acceptance-commitment (MAC) approach. New York: Springer Publishing Company.

Gooding, A. y Gardner, F.L. (2009). An empirical investigation on the relationship between mindfulness, pre shot routine, and basketball free throw percentage. Journal of Clinical Sports Psychology, 3, 303-319.

Hardy, L., Jones, G. y Gould, D. (1996). Understanding psychological preparation for sport: Theory and practice of elite performers. New York: Wiley.

Hayes, S. C. (2004). Acceptance and commitment therapy, relational frame theory, and the third wave of behavioral and cognitive therapies. Behavior 
therapy, 35, 639-665.

Hayes, S. C., Strosahl, K. y Wilson, K. G. (1999). Acceptance and commitment therapy: An experiential approach to behavior change. New York: Guilford Press.

Hayes, S.C., Strosahl, K.D. y Wilson, K.G. (1999). Acceptance and Commitment Therapy: An experiential approach to behavior change. New York: Guilford Press.

Kabat-Zinn, J. (1990). Full catastrophe living. Nueva York: Delacorte.

Kabat-Zinn, J. (2003). Vivir con plenitud las crisis. Cómo utilizar la sabiduría del cuerpo y la mente para afrontar el estrés, el dolor y la enfermedad. Barcelona: Kairós.

Kaufman, K. A. y Glass, C. R. (2006). Mindful Sport Performance Enhancement: A treatment manual for archers and golfers. Unpublished manuscript, The Catholic University of America, Washington, DC.

Kaufman, K. A., Glass, C. R. y Arnkoff, D. B. (2009). Evaluation of Mindful Sport Performance Enhancement (MSPE): A new approach to promote flow in athletes. Journal of Clinical Sport Psychology, 4, 334-356.

Kaufman, K. A., Glass, C. R. y Pineau, T. R. (2012). Mindful Sport Performance Enhancement: A treatment manual for long-distance runners. Manuscript in preparation, The Catholic University of America, Washington, DC.

Lee, K. y de Bondt, R. (2005). Total archery. Republic of Korea: Samick Sports Co., Ltd.

Lutkenhouse, J. M. (2007). The case of Jenny: A freshman collegiate athlete experiencing performance dysfunction. Journal of Clinical Sport Psychology, 1, 166-180.

Lutkenhouse, J., Gardner, F. L. y Morrow, C. (2007). A randomized controlled trial comparing the performance enhancement effects of Mindfulness-Acceptance-Commitment (MAC) performance enhancement and psychological skills training procedures. Manuscript in preparation.

Mañas, I. (2007). Nuevas terapias psicológicas: La tercera ola de terapias de conducta o terapias de tercera generación. Gaceta de Psicología, 40, 26-34.

Meichenbaum, D. (1977). Cognitive behaviour modification: An integrative approach. New York: Plenum.

Mellalieu, S. D., Hanton, S. y Fletcher, D. (2006). A competitive anxiety review: Recent directions in sport psychology research. En S. Hanton \& S. D. Mellalieu (Eds.), Literature reviews in sport psychology, (pp. 1-45). New York: Nova Scienc Publishers, Inc.

Pineau, T. R., Kaufman, K. A. y Glass, C. R. (2012). Effects of Mindful Sport Performance Enhancement (MSPE) on running performance and body image: Does self-compassion 52 make a difference? Manuscript in preparation, The Catholic University of America, Washington, DC.

Purdon, C. (1999). Thought suppression and psychopathology. Behaviour Research and Therapy, 37, 1029-1054.

Rassin, E., Merckelbach, H. y Muris, P. (2000). Paradoxical and less pradoxical 
effects of thought suppression. A critical review. Clinical Psychology Review, 20, 973-995.

Rotella, B. y Cullen, B. (2004). The golfer's mind: Play to play great. New York: Free Press.

Schwanhausser, L. (2009). Application of the mindfulness-acceptance-commitment (MAC) protocol with an adolescent springboard diver. Journal of Clinical Sport Psychology, 4, 377-395.

Segal, Z.V., Williams, J.M.G. y Teasdale, J.D. (2002). Mindfulness-based cognitive therapy for depression: A new approach to preventing relapse. New York: The Guilford Press.

Thompson, R. W., Kaufman, K. A., De Petrillo, L. A., Glass, C. R. y Arnkoff, D. B. (2011). One year follow-up of mindful sport performance enhancement (MSPE) for archers, golfers, and long-distance runners. Journal of Clinical Sport Psychology, 5, 99-116.

Williams, J.M. y Straub, W.F. (2010). Sport Psychology: Past, Present, Future. En: J.M. Williams (Ed.). Applied Sport Psychology (6th ed), (p. 1-17). New York: McGrawHill.

Wolanin, A. T. (2005). Mindfulness-Acceptance-Commitment (MAC) based performance enhancement for Division I collegiate athletes: A preliminary investigation (Doctoral dissertation, La Salle University, 2003). Dissertation Abstracts International-B, 65, pp. 3735-3794. 